7

8

9

\title{
Global Response to Local lonospheric Mass Ejection
}

\author{
T. E. Moore ${ }^{1}$, M.-C. Fok ${ }^{1}$, D. C. Delcourt ${ }^{2}$, S. P. Slinker ${ }^{3}$, and J. A. Fedder ${ }^{4}$
}

1. NASA Goddard SFC, Code 670, Greenbelt MD 20771 USA

2. Centre d'études des l'Environnements Terrestre et Planétaires, St.-Maur, FRANCE

3. Naval Research Laboratory, Washington DC 20375 USA

4. Leading Edge Technology, Inc., Alexandria, VA 22301 USA

Submitted to J. Geophys. Res.: 30 April 2010; Revised 12 July 2010

\section{Abstract}

We revisit a reported "Ionospheric Mass Ejection" using prior event observations to guide a global simulation of local ionospheric outflows, global magnetospheric circulation, and plasma sheet pressurization, and comparing our results with the observed global response. Our simulation framework is based on test particle motions in the LyonFedder-Mobarry (LFM) global circulation model electromagnetic fields. The inner magnetosphere is simulated with the Comprehensive Ring Current Model (CRCM) of Fok and Wolf, driven by the transpolar potential developed by the LFM magnetosphere, and includes an embedded plasmaspheric simulation. Global circulation is stimulated using the observed solar wind conditions for the period 24-25 Sept 1998. This period begins with the arrival of a Coronal Mass Ejection, initially with northward, but later with southward interplanetary magnetic field. Test particles are launched from the ionosphere with fluxes specified by local empirical relationships of outflow to electrodynamic and particle precipitation imposed by the MHD simulation. Particles are tracked until they are 
1 lost from the system downstream or into the atmosphere, using the full equations of

2

3

4

5

6

7

8

9

10

11

12

13

14

15

16

17

18

19

20

21

22 
 \\ Introduction}

In this paper, we revisit the "ionospheric mass ejection" event reported by Moore et al. [1999], as a response to a coronal mass ejection that arrived at the beginning of 25 Sept. 1998. The observed event was characterized by a substantial increase in the dayside outflow of auroral wind $\mathrm{O}^{+}$, observed by the Polar/TIDE instrument, mainly in the polar lobes. Heavy molecular ions were also observed to have been produced by the Polar/TIMAS mass analyzer. Pre-existing ion outflows were observed to respond dramatically to the passage of the shock leading the CME through the polar ionosphere [Cladis et. al. 2000]. And it was suggested that the outflow increases from the dayside ionosphere were enhanced by the increased solar wind dynamic pressure, following DE1/RIMS observations reported earlier by Pollock et al. [1990]. Strangeway et al., [2000] studied the same event using FAST observations of lower altitude outflows and suggested that they appeared to be driven by enhanced DC Poynting and electron precipitation fluxes into the ionosphere. Subsequent observations have reinforced that ionospheric outflows respond to enhancements of the solar wind dynamic pressure [Elliott et al., $2001 ; 2007]$. This is particularly dramatic when the dynamic pressure increases abruptly with the arrival of the interplanetary shock at the leading edge of a Coronal Mass Ejection, as occurred, for example, on 24 Sept 1998. A fraction of the solar energy release that created the CME is deposited in the ionosphere, sufficient to eject a portion of the topside plasma from Earth's ionosphere.

Moore et al. [1999] reported an empirical relationship between the solar wind dynamic pressure and the outflow of ionospheric plasma, suggestive of a direct link between solar wind energy and mechanisms responsible for ionospheric heavy ion 
outflows. Those authors also reported a lack of correlation between ionospheric outflows and the orientation of the interplanetary magnetic field, a result that was puzzling in view of the importance of magnetic coupling between the IMF and the geomagnetic field, via reconnexion.

Strangeway et al. [2005] investigated this puzzle using the FAST data set for the same event in Sept 1998, which permitted detailed studies within the outflow generation region. They found that the local outflow flux was highly correlated with the local Poynting flux of electromagnetic energy into the ionosphere, and with the density of electron precipitation into the ionosphere. For FAST observations near $4000 \mathrm{~km}$ altitude, the outflow flux was found to scale with (Poynting Flux) $)^{1.3}$ and with (Hot e- density) ${ }^{2.2}$. These results clearly pointed to a combination of electromagnetic and kinetic energy deposition as being responsible for the observed outflows. The flow path for solar wind energy into the ionosphere is plausibly associated with the presence of an energetic solar wind flow, which is then linked into the magnetosphere and ionosphere along open flux tubes created by dayside reconnexion, leading to the transmission of energy and hot plasma into the topside ionosphere with a spatial distribution that must depend on the distribution of dayside reconnexion.

The outflow presumably results from a combination of ion heating derived from the DC Poynting flux and ambipolar potential enhancement by the superthermal electron population produced in the topside ionosphere by electron precipitation. The former most likely derives from the strongly driven convection of plasma through the neutral gas, while the latter derives from zero current requirement to retard fast electron escape sufficiently to be balanced by ion escape. In a companion paper [Moore and Khazanov, 
this issue], it is shown that the Strangeway relations can be reproduced by "generalized Jean's escape" of transversely accelerated ions that exhibit power law velocity distributions scaled with the local convection speed. Such distributions are expected to result from the relaxation of toroidal pick-up ion distributions, in the case of ion speeds much less than the local Alfvén speed. The generalizations of Jeans' escape include the assumption of nonthermal velocity distributions escaping from the combination of ambipolar, and centrifugal potentials, as well as gravity.

Peroomian [2006] studied this event using their Large Scale Kinetics method, driving ionospheric outflows from the cusp region according to the relationship suggested by Moore et al. [1999], finding that significant $\mathrm{O}^{+}$circulation and acceleration resulted, consistent with the results of Nosé [2005]. Comparisons were also made with Kistler [2005], with the finding that plasma sheet and ring current densities were reasonably consistent with those observations. The $\mathrm{O}^{+}$spatial distribution was simulated to be skewed toward dusk early in the storm, but later became skewed toward dawn. The peak $\mathrm{O}^{+}$ring current was found to be relatively small at $10 \mathrm{nT}$ at storm peak. Moreover, the $\mathrm{O}^{+}$ ions were quickly lost from the inner magnetosphere producing a rapid decay of ring current intensity. Peroomian [2007] further studied this event to provide a comparison of solar wind and ionospheric contributions to the hot plasma. The IMF Bz variations for this event introduce corresponding variations in magnetospheric convection and access of ionospheric outflows to the magnetotail. $\mathrm{O}^{+}$was initially a $20 \%$ contributor to the ring current, but later came to dominate over the solar wind protons, in both number density and energy density. 
The purpose of the present paper is to re-investigate the above scenario using the new empirical outflow relationships that have come to light from recent work. We incorporate models of polar wind $\mathrm{H}^{+}$outflows driven by solar UV illumination [Su et al., 1998], and a scaling of auroral wind $\mathrm{O}^{+}$outflows driven empirically [Strangeway et al. 2005] by local Poynting and kinetic fluxes into the ionosphere. We include a model of the plasmasphere that produces reasonable quantitative transport and resultant spatial distributions of plasmaspheric material [Ober et al., 1997], as reported by Moore et al., [2008]. The Comprehensive Ring Current Model of Fok, Wolf, et al. [2001] is embedded within the global magnetospheric simulation of Lyon, Fedder, and Mobarry [Lyon et al., 2004].

These models collectively comprise our Global Ion Kinetic simulation of the magnetospheric response to solar wind conditions. Here we apply them to the specific 2425 Sept. 1998 event, including dayside reconnexion, the generation of field aligned currents that drive ionospheric convection, and thus the spatial distribution and temporal variation of the DC Poynting flux into the ionosphere. The simulated distribution of hot plasma density is used to estimate precipitating electron density. In combination with the Poynting flux, this permits assessment of ionospheric outflow using the Strangeway [2005] empirical relations, which in turn are based on FAST in situ observations of the simulated event. The models calculate large numbers (millions) of ionospheric ion trajectories, tracking them throughout the magnetosphere. This permits the computation of both kinetic and bulk characteristics of the ionospheric plasmas throughout the entire magnetosphere, serving as inputs to the inner magnetospheric ring current simulation. We thus investigate whether the locally observed, empirical $\mathrm{O}^{+}$outflows produce a global 
1 response that is consistent with observations and prior simulation work base on more a global outflow assessment.

In the next sections we describe the various models and their linkage, the ionospheric outflow distribution, the solar and ionospheric plasma circulation throughout the magnetosphere, and the global impact on storm time ring current as compared with the observed ring current. Finally, we compare our results with observations and with an independent investigation of this event [Peroomian et al., 2006] in which the global outflow was determined from the Moore et al. [1999] correlation of outflow with solar wind dynamic pressure, rather than the Strangeway [2005] relations.

\section{Model Descriptions}

Figure 1 summarizes our simulation approach. The Lyon-Fedder-Mobarry (LFM) ideal MHD global circulation model [Lyon et al., 2004] provides the framework for computing ion trajectories in specified electromagnetic fields throughout the outer magnetosphere, including the magnetosheath, bow shock and beyond. Gravity is also included since the lowest energy ions are affected. The LFM simulation tracks only a solar wind plasma fluid, with the inner boundary assumed to be magnetically-coupled to an ionosphere of specified conductivity. In this work, particular attention was given to the computed hot plasma fluid parameters, electromagnetic fields, and linking current density on an inner boundary of the simulation space, because these were used to drive ionospheric outflow responses.

The ionospheric conductance model consisted of a component due to solar illumination and a component calculated from auroral precipitation [Slinker et al., 1999]. Equinox conditions were assumed and the peak dayside conductance was about 10 mho. 
1 The dimensions of the LFM simulation space extended from +24 to $-300 \mathrm{RE}$ in $\mathrm{X}_{\mathrm{SM}}$ and $\pm 90 \mathrm{RE}$ in $\mathrm{Y}_{\mathrm{SM}}$ and $\mathrm{Z}_{\mathrm{SM}}$. The inner boundary of the LFM simulation was at $3.2 \mathrm{RE}$ radius. The particle simulation space ranged from +24 to $-70 \mathrm{RE}$ in $\mathrm{X}_{\mathrm{GSM}}$, and from \pm 30 $\mathrm{R}_{\mathrm{E}}$ in $\mathrm{Y}_{\mathrm{GSM}}$ and $\mathrm{Z}_{\mathrm{GSM}}$. WIND observations of the solar wind interval having large variations are shown in Figure 2. For this event, we simulated a 30-hour period of response, as shown in Figure 3.

Within the LFM simulation of solar wind plasmas, as previously reported [Moore et al. 2005; 2007; 2008], we model the behavior of polar wind, auroral wind and plasmaspheric wind plasmas, as well as their supply of plasma to the ring current for this event [Fok et al., 2006], using single particle Monte Carlo calculations. We treat the polar wind protons as flowing outward along magnetic flux tubes everywhere outside the plasmapause. We treat the auroral wind as an $\mathrm{O}^{+}$outflow that is embedded within the polar wind, and we accelerate both polar wind protons and auroral wind oxygen according to parallel potential drops derived from the [Lyons, 1981] version of the Knight relationship and driven by the intensity of upward coupling currents imposed by the LFM simulation at the ionospheric boundary. We treat the plasmasphere as an emissive surface through which proton flux drifts as determined by the modeled density on the surface and convective flow speed across it, as described in the papers cited above. Briefly, ion starting points were randomly distributed in latitude and local time on the ionospheric and plasmaspheric boundaries. Initial velocities were randomly selected from a range matching observed ion thermal speeds, which also scale with the Poynting flux in the auroral wind context, according to Strangeway [personal communication]. Initial pitch angles were selected randomly from a range corresponding to the local ratio of 
thermal to parallel flow speeds. Initial gyro-phases were selected randomly from the full range of $360 \mathrm{deg}$. around the local convection flow speed, as specified by the global simulation. The trajectories were computed and recorded in a database until the particles either entered the atmosphere or escaped from the simulation space, using the full equations of motion and a computation process described by Delcourt et al. [1993].

Particle weightings follow the method of Moore et al. [2005]. Briefly, for each particle in a particular simulation spatial bin, the particle velocity and transit time for that bin are calculated. For a particle passing through a particular bin, the particle contribution to density in this bin is the transit time $(\mathrm{T})$, divided by the bin volume $\left(\mathrm{L}^{3}\right)$, times the source fluence $\left(\mathrm{T}^{-1}\right)$ for ions of the specified velocity. Source fluence $\left(\mathrm{T}^{-1}\right)$ for each ion is computed directly from the source flux (or density $\mathrm{x}$ flow; $\mathrm{L}^{-2} \mathrm{~T}^{-1}$ ) of the source plasma across the source boundary, multiplied by the area $\left(\mathrm{L}^{2}\right)$ of the source, divided by the number of ions launched from that area (total source area / \# of ions launched).

Into the LFM simulation context is embedded the Comprehensive Ring Current Model [Fok et al., 2001], which includes the nested plasmasphere model adapted from that of D. M. Ober [1997], . The CRCM solves the bounce averaged Vlasov equation for inner magnetospheric convection self-consistently with electrodynamic coupling to the ionosphere, driven by the LFM simulation for transpolar potential. CRCM requires that flux tubes are supplied from outside the simulation space with specified plasma content and magnetic geometry. CRCM was supplied with plasmas as calculated from the LFM simulation for solar wind protons, combined with ionospheric proton and oxygen plasmas computed from the particle trajectory method. As flux tubes convect into the space according to the imposed external convection specification, they begin to fill owing to 
$1 \quad$ local plasmaspheric ionospheric proton outflows that are computed by the Ober [1997] method, so as to shut down realistically as the flux tubes fill up. The result is that flux tubes that persist inside the model space become filled within an appropriate time constant that is set by the volume of the flux tube and the assumed strength of the ionospheric outflow flux.

The approach we have described above invokes an ionosphere and plasmasphere that are driven by the convection imposed upon the magnetosphere by the LFM description of the solar wind interaction, with dissipative flow loading by ionospheric drag via field aligned currents with associated Poynting flux and precipitation. The simulation includes co-rotation and produces typical plasmaspheric plumes when magnetospheric convection is enhanced. We make a transition to single particle motions above the ionosphere, outside the plasmasphere proper and beyond the CRCM boundary, so that we can then track ionospheric outflows and plasmaspheric plumes through the structured and variable fields of the outer magnetosphere, its boundary layers, and account for the full nonadiabatic behavior of the particles, using a realistic model of those structured fields. As a reasonable approximation of the fields, we use the ideal MHD global simulation. This approach involves only one way coupling from the solar wind plasmas to the ionosphere proper and does not allow the outer magnetosphere simulation to react to the presence outflowing ionospheric plasmas. On the other hand, the CRCM does compute selfconsistent convection electric fields within the inner magnetosphere, including current flows linking the ionospheric F region. These fields do respond to the presence or absence of plasma as determined by the boundary conditions for the CRCM domain, or inner magnetosphere. 
In the present work we compute the contribution to the ring current of the solar, polar, auroral, and plasmaspheric winds for a specific CME event that leads to the substantial outflow of ionospheric heavy ion plasma previously referred to as an "ionospheric mass ejection," occurring in response to arrival of a coronal mass ejection at Earth.

\section{Circulation Results}

\section{Solar Wind}

The upstream solar wind conditions for this event have been published previously [Moore et al., 1999, Figure 1; Peroomian et al. 2006, Figure 1]. The latter are reproduced here in Figure 2. As the driver of magnetospheric and ionospheric responses, they may be briefly summarized as follows: The period preceding the CME arrival was characterized by dynamic pressure between $1-1.5 \mathrm{nPa}$ with an interplanetary magnetic field of about 10 nT dominated by positive GSM y-component (away sector). A substantial dynamic pressure jump occurred with arrival of the interplanetary shock leading the CME at approximately $24: 00 \mathrm{hrs}$ on 24 Sep 1998 . The peak dynamic pressure reached approximately $6 \mathrm{nPa}$. With arrival of the shock, the IMF turned northward to a clock angle of about $45 \mathrm{deg}$, and intensified to over $30 \mathrm{nT}$. During this period a positive Dst perturbation, or sudden commencement, was observed. During the first hour after arrival, the IMF rotated to nearly southward, and Dst rapidly dropped to $-150 \mathrm{nT}$ and then further to $-200 \mathrm{nT}$ at the peak phase of the storm, about 8-10 hours after CME arrival.

Figure 3 illustrates the simulated MHD conditions in the magnetosphere during the main phase of the storm, near the maximum time rate change of Dst. Here it can be seen that the nightside near-Earth plasma sheet pressure is actually somewhat larger than that of the incident solar wind at that time. Another noteworthy feature is the evacuation of 
1 the polar lobe regions, which is typical under southward IMF conditions owing to rapid convection over the poles and consequent centrifugal acceleration.

\section{Polar, Auroral Winds}

We next summarize the results for polar wind and auroral wind behavior for this storm period. As reported in our previous studies [Moore et al. 2005; 2007], we simulate polar wind outflows as being independent of solar wind or ionospheric conditions, with local outflow flux that depends mainly on solar zenith angle [Su et al., 1998], with fluxes dropping by an order of magnitude on the dark side of the terminator. However, protons are substantially accelerated in auroral regions with upward parallel current flows as given by the MHD simulation, using the Lyons [1981] version of the Knight relationship.

Such parallel acceleration, combined with generally faster convection speeds and associated centrifugal acceleration effects, leads to rapid escape of auroral-accelerated polar wind plasmas through the lobes of the magnetotail or the flanks of the magnetosphere. The polar wind results illustrate many familiar features of magnetospheric plasma dynamics for this storm period. However, these earlier studies indicated a modest contribution to the hot plasma pressure of the inner magnetosphere and the ring current in particular, and that finding is reproduced here.

Polar wind particle weightings were guided by observations of flux and velocity [ $\mathrm{Su}$ et al., 1998], and produced realistic outflows into the lobes of low density, low energy outflows. A substantial fraction of the outflowing polar wind was found to enter the magnetosheath via high latitude reconnexion and to escape from the system downstream, as shown in Figure 5. This is particularly true for "auroral" polar wind, that is, polar wind that has become part of the auroral outflow by virtue of its acceleration by auroral effects. 
1 However it is the auroral wind of outflowing heavy $\mathrm{O}^{+}$ions that is the focus of the present study. Our implementation of the Strangeway [2005] outflow scalings is used to produce local $\mathrm{O}^{+}$outflows based on local DC Poynting flux and electron precipitation according to the same method used in Moore et al., [2007, 2008], and briefly described above. Figure 6 illustrates this in the form of a snapshot of the outflow drivers and the response to them, in two rows of polar plots of the ionospheric distribution at a particular time during the event.

The temporal behavior of ionospheric outflows produced in our simulation is illustrated by Figure 7, where the global fluence is assessed and compared with that found in an earlier simulation study of this event. Dst is plotted in this figure principally as a guide to the overall development sequence of the storm, along with the vertical line indicating the arrival of the CME at 24:00 UT on 24 Sept. It's clear that the solar wind interaction was already appreciable prior to the arrival of the CME. With mainly positive IMF By and a few nPa of dynamic pressure, the global outflow was already approaching $1 \times 10^{27} \mathrm{O}^{+} / \mathrm{sec}$. We previously have benchmarked our approach and shown that it is is comparable with the Yau and André [1997] statistical results for typical conditions, but exceeds those limits for large events, so this seems reasonable. The arrival of the CME drove that value up by another factor of $\sim 2$, producing high fluence that persisted throughout the event and then trailed off somewhat more slowly than the Dst magnitude did late in the event. Peak local outflow fluxes in the cusp region correspondingly increased early in the period immediately after 20:00, and then increase another factor of 3 or more at $24: 00 \mathrm{UT}$. 
The simulation performed by Peroomian [2006] simulated a shorter period beginning at about 23:00 UT and ending at the peak phase of the storm, about 08:00 UT on 25 Sept. Outflow was considered to flow in a restricted region from 11-13 hours of MLT, and 70$85^{\circ}$ MLat. The $\mathrm{O}^{+}$flux from this region was driven by the relationship between local fluxes and solar wind dynamic pressure suggested by Moore et al., [1999]. Thus the total fluence numbers are not strictly comparable, but do show some corresponding features. This is understandable, since the present simulation considers and Figure 7 sums over the global ionosphere, as excited by local conditions determined from the LMF boundary conditions.

Substantial auroral wind was found to be present at high altitudes in the magnetosphere during the initial period of the event, prior to the arrival of the CME with its enhanced dynamic pressure and fluctuating IMF. This is consistent with observations of the event reported by Moore et al., [1999], and the results obtained by Cladis et al., [2000], who found that the CME shock arrival accelerated pre-existing $\mathrm{O}^{+}$plasmas present in the polar cap and lobe observed by Polar, from $10 \mathrm{~s}$ of eV to over $1 \mathrm{keV}$ of streaming energy. Enhanced auroral wind was also driven by the enhanced auroral zone coupling of energy and solar wind plasmas that resulted when the CME arrived, regardless of the IMF sense being northward initially. This auroral wind was much more confined to the inner magnetosphere than the polar wind protons, and a relatively small amount escaped into the dayside magnetosheath and downstream for this event. However, a large amount of auroral wind was supplied to the near Earth plasma sheet, and it then convected back through the inner magnetosphere, becoming part of the ring current. This is illustrated in Figure 8. 


\section{Global Impact Results}

With the solar wind supply of plasma to the inner magnetosphere (Figure 4) and the polar wind supply (Figure 5) we now look at the aggregate supply of auroral wind $\mathrm{O}^{+}$ plasma to the inner magnetosphere, as shown in Figure 8. Here we see clearly in the YZ and $\mathrm{XZ}$ planes the polar outflows at low pressure $(0.01$ to $0.1 \mathrm{nPa})$, and their accumulation into the plasma sheet, especially evident in the $\mathrm{YZ}$ and $\mathrm{XY}$ planes. In the latter, a substantial accumulation can be seen to encircle the Earth, with pressure approaching that of the solar wind protons, in the range from $1-10 \mathrm{nPa}$. The pressure of polar wind proton plasmas is much lower, in the range 0.01 to $0.1 \mathrm{nPa}$. Thus, we can anticipate that the principal sources to the ring current and inner magnetosphere to be solar wind protons and auroral wind oxygen.

To illustrate the auroral wind participation in the inner magnetospheric plasma pressure distribution, we show in Figure 9 the relevant pressure distributions, at a point near the maximum of the Dst magnitude, at about $-200 \mathrm{nT}$, for both $\mathrm{O}^{+}$and solar wind protons. Clearly both are contributing significantly to the inner magnetospheric pressure distribution at the storm peak.

Next we examine the total/integral plasma energy content of the inner magnetosphere, defined as the region inside the $\mathrm{CRCM}$ simulation boundary at $\mathrm{L}=8$. The result of integrating total particle content over this volume is shown in Figure 10. An increase in solar wind $\mathrm{H}^{+}$content inside the inner magnetosphere clearly results. A smaller but comparable increase in the auroral wind $\left(\mathrm{O}^{+}\right)$content also occurs, albeit with a delay relative to the solar wind content of perhaps 6 hours. The former is relatively prompt owing to its continuous presence in the magnetospheric lobes and magnetotail, while the 
1 latter is somewhat delayed from its initial transient release by transport time from the ionosphere through the lobes and plasma sheet and back to the inner magnetosphere after energization. The total plasma pressure including both contributions has a shape that matches reasonably well the growth phase of Dst. However, the decay phase is proceeding considerably more slowly in the simulation than in the observed Dst. We treat this point in the discussion below.

\section{Discussion}

Here, we have brought our Global Ion Kinetic method to bear on the simulation of this event, with results that should be compared with those of Peroomian [2006, 2007]. The principal difference is that we have expanded the description of ionospheric outflows by incorporating the local outflow scalings of Strangeway et al. [2005] over the entire polar cap. These respond to local conditions generated at the inner boundary of the MHD outer magnetosphere simulation. Reference is made to a companion paper [Moore and Khazanov, this issue] for a derivation of the local scaling relations based on Poynting flux and kinetic precipitation conditions derived locally from a global MHD simulation. In addition, we also implement an inner magnetosphere or ring current simulation that contains full drift physics and generally tends to produce more realistic ring current magnitudes than MHD simulations of the inner magnetosphere. Thus this study can be regarded as a test of the use of global ionospheric outflows driven by local conditions within a simulation of the solar wind-magnetosphere interaction, with a more realistic inner magnetospheric simulation of a real magnetospheric event.

The period prior to the CME arrival was already active in the sense of having significantly elevated dynamic pressure, with By positive and substantial. Thus 
1 ionospheric outflows as produced by the Strangeway relations was already moderate prior to the arrival of the CME shock, but increased substantially with its arrival. Moreover, substantial acceleration and pressurization of the pre-existing outflows occurred with its arrival, enhancing plasma pressure throughout the magnetosphere and particularly at the boundary of the inner magnetosphere. Then Bz turned southward and produced enhanced deep convection through the inner magnetosphere that enhanced the pressures within that region, commensurate with the observed value of Dst that was reached in this storm period. We found that early in the storm, Dst was solar wind proton dominated, but after several hours, $\mathrm{O}^{+}$became comparable in pressure contribution as protons faded and $\mathrm{O}^{+}$increased. This is consistent with the results obtained by Nosé [2005], who found overwhelming $\mathrm{O}^{+}$dominance for substantially larger storms than the one studied here.

Overall, the simulation produces most features of the observed storm. One notable exception is the rapid decay of the observed ring current after the storm peak. The simulated ring current is appreciably slower to decay. This indicates either deficient loss mechanisms or an excessive continuing source of $\mathrm{O}^{+}$outflow during the recovery phase of the storm. Our loss mechanisms have been validated in other simulations and are believed to be realistic. The discrepancy could result from an excessive response of the outflow flux scalings to electron precipitation, which would be expected to be substantial over large areas of the polar regions and extending down to lower latitudes during the recovery phase. During the recovery phase, we would expect Poynting flux energy inputs to have subsided substantially. This suggests that the outflow scalings we have used may not be accurate in regions where these two parameters have a different relationship than 
on the dayside from which the observations originated that were used as a basis for them [Strangeway et al., 2005]. In the future, we plan to study in more detail the coupling between Poynting and kinetic energy inputs to ionospheric $\mathrm{O}^{+}$outflows, and to revisit this type of event to test this hypothesis.

We again find in our GIK simulation that the initial energy of ionospheric plasma ions in the magnetospheric system seems to be unimportant to their ultimate fate. Of the fast and light protons of the polar wind, only a small fraction will be circulated into the interior magnetosphere. The bulk of them will be picked up by the solar wind to like velocities and escape downstream as soon as they convect onto open or boundary layer flux tubes. On the other hand, most of the slow and heavy $\mathrm{O}^{+}$ions will remain relatively close to the Earth and escape into the magnetosheath to a lesser degree, and at higher energy. They will tend to make multiple trips around the magnetospheric circulation path, gaining energy until they are finally fast enough to behave like protons and escape into the magnetosheath. Thus, while trapping by gravity ceases as soon as $\mathrm{O}^{+}$ions are accelerated above $10 \mathrm{eV}$, their large mass and slow velocity at given energy remains a significant factor in retaining them close to Earth. Conversely, geogenic protons reach the magnetosheath more readily, mixing with solar protons there, or escaping into the solar wind at any opportunity presented by open flux tubes.

Our results must be qualified by the knowledge that we have simulated ionospheric outflows through the outer magnetosphere using test particles in MHD fields that are consistent with negligible presence of ionospheric pressure. However, within the CRCM inner magnetospheric simulation, ionospheric plasmas are dynamic elements that do alter the global convection pattern. However, these results could change, perhaps significantly, 
1 when they are superseded by multi-fluid or multi-species studies that compute the influence of ionospheric plasma inertia and pressure in the outer magnetosphere.

\section{Conclusions}

Based upon our GIK simulations of dynamic 3D magnetospheric fields, the full trajectories of solar and ionospheric ions in those fields, and the kinetic behavior of inner magnetospheric plasmas, we conclude the following:

Simulations of a well-observed event are reproducible by independent studies that achieve comparable qualitative agreement with observations.

A more global and locally responsive outflow formulation has been used in the present simulations, as an alternative to an approach based on the Moore [1999 GRL] scaling of outflow with solar wind Pd or its variations.

Not surprisingly, the total fluence of ionospheric plasma is substantially greater when global outflows are assessed. With the Strangeway et al. [2005] local outflow scaling based on Poynting flux and electron precipitation, these more extensive outflows produce a somewhat greater ionospheric participation in the plasma sheet and ring current that was found by Peroomian [2006, 2007] for an ionospheric source with comparable fluxes, but confined to the dayside cusp region.

Reasonable qualitative agreement is found with observed global measures of ring current during growth and peak phases, suggesting that the dayside source makes a large contribution to them.

The decay phase of the storm did not proceed as fast in the simulations as in the observations. However, we expect that this discrepancy can be corrected by a more 
accurate treatment of wave particle interactions and resultant pitch angle diffusion, with enhanced loss rates from the ring current.

\section{Acknowledgements}

We thank Manuel Buenfil and Robert Wiegand, who through their mastery of mathematics, programming, database, and visualization, made this work possible. We acknowledge support from the NASA Heliophysics Division through the ROSES program under WBS 936723.02.01.03.82, and NASA's High Performance Computing Program.

\section{References}

Cladis, J. B., H. L. Collin, O. W. Lennartsson, T. E. Moore, W. K. Peterson, and C. T. Russell (2000), Observations of centrifugal acceleration during compression of magnetosphere, Geophys. Res. Lett., 27(7), 915-918.

Delcourt, D.C., J.A. Sauvaud, and T.E. Moore (1993), Polar wind ion dynamics in the magnetotail, J. Geophys. Res. 98(A6), 9155.

Elliott, H.A., Comfort, R.H., Craven, P.D., Chandler, M.O., Moore, T.E. (2001), Solar wind influence on the oxygen content of ion outflow in the high-altitude polar cap during solar minimum conditions. J. Geophys. Res., 106, 6067-6084.

Elliott, H. A, J.-M. Jahn, C. J. Pollock, T. E. Moore, and J. L. Horwitz (2007), Journal of Atmospheric and Solar-Terrestrial Physics 69, pp.1541-1555.

Fok, M.-C., R. A. Wolf, R.W. Spiro, and T. E. Moore (2001), Comprehensive computational model of Earth's ring current, J. Geophys. Res. 106, A5, p.8417, 1 May 2001. 
1 Fok, M.C., Moore, T.E., Brandt, P.C., Delcourt, D.C., Slinker, S.P., Fedder, J.A., (2006), Impulsive enhancements of oxygen ions during substorms. Journal of Geophysical Research.

Kistler, L. M., et al. (2005), Contribution of nonadiabatic ions to the cross-tail current in an O+ dominated thin current sheet, J. Geophys. Res., 110, A06213, doi:10.1029/2004JA010653.

Lyon, J.G., Fedder, J.A., Mobarry, C.M. (2004), The Lyon-Fedder-Mobarry (LFM) global MHD magnetospheric simulation code. Journal of Atmospheric and SolarTerrestrial Physics 66 (15\&16), 1333-1350.

Lyons, L.R., 1981. The field-aligned current versus electric potential relation and auroral electrodynamics. In: Akasofu, K. (Eds.), Physics of Auroral Arc Formation. Geophysics Monograph 25, American Geophysics Union, p. 252.

Moore, T. E., W. K. Peterson, C. T. Russell, M. O. Chandler, M. R. Collier, H. L. Collin, P. D. Craven, R. Fitzenreiter, B. L. Giles, and C. J. Pollock (1999), Ionospheric mass ejection in response to a CME, Geophys. Res. Lett., 15, 2339-2342.

Moore, T. E., M.-C. Fok, M. O. Chandler, C. R. Chappell, S. P. Christon, D. C. Delcourt, J. Fedder, M. Huddleston, M. Liemohn, W. K. Peterson, and S. Slinke8 (2005), Plasma sheet and ring current formation from solar and polar wind sources. J. Geophys. Res., 110, A02210.

Moore, T. E., M.-C. Fok, D.C. Delcourt, S.P. Slinker, J.A. Fedder (2007), Global aspects of solar wind-ionosphere interactions, Journal of Atmospheric and Solar-Terrestrial Physics 69, pp.265-278. 
1 Moore, T. E., M.-C. Fok, D. C. Delcourt, S. P. Slinker, and J. A. Fedder (2008), Plasma plume circulation and impact in an MHD substorm, J. Geophys. Res., 113, A06219, doi:10.1029/2008JA013050.

Moore, T.E., and G. V. Khazanov, 2010, Mechanisms of Ionospheric Mass Escape, J. Geophys. Res., this issue.

6

7

Nosé, M., S. Taguchi, K. Hosokawa, S. P. Christon, R. W. McEntire, T. E. Moore, and M. R. Collier (2005), Overwhelming $\mathrm{O}^{+}$contribution to the plasma sheet energy density during the October 2003 superstorm: Geotail/EPIC and IMAGE/LENA observations, J. Geophys. Res., 110, A09S24, doi:10.1029/2004JA010930.

Ober, D. M, J. L. Horwitz, and D. L. Gallagher, (1997), Formation of density troughs embedded in the outer plasmasphere by subauroral ion drift events, J. Geophys. Res., $102,14,595$.

Pollock, C. J., M. O. Chandler, T. E. Moore, J. H. Waite Jr., C. R. Chappell and D. A. Gurnett, 1990, A survey of upwelling ion event characteristics, J. Geophys. Res. 95, 18969.

Peroomian, V., M. El-Alaoui, M. A. Abdalla, and L. M. Zelenyi (2006), Dynamics of ionospheric $\mathrm{O}^{+}$ions in the magnetosphere during the 24-25 September 1998 magnetic storm, J. Geophys. Res., 111, A12203, doi:10.1029/2006JA011790.

Peroomian, V., Mostafa El-Alaoui, Maha Ashour Abdalla, Lev M. Zelenyi (2007), A comparison of solar wind and ionospheric plasma contributions to the September 24 25, 1998 magnetic storm, JASTP 69 (2007) 212-222. 
1 Slinker, S.P., et al., 1999, Comparisons of global MHD simulations with AMIE

2

3

4

5

6

7

8

9

10

11

12

13

14

15

16

17

18

19

20

21

22 5 7 simulations for the events of May 19-20, 1996. Journal of Geophysical Research 104, 28379.

Strangeway, R. J., C. T. Russell, C. W. Carlson, J. P. McFadden, R. E. Ergun, M. Temerin, D. M. Klumpar, W. K. Peterson, and T. E. Moore (2000), Cusp field-aligned currents and ion outflows, J. Geophys. Res., 105, 21,129-21,142.

Strangeway R. J., et al., 2005, Factors controlling ionospheric outflows as observed at intermediate altitudes, J. Geophys. Res., A03221.

Su, Y.-J., et al., (1998), Polar wind survey with TIDE/PSI suite abroad POLAR. Journal of Geophysical Research 103, 29,305.

Yau, A.W., André , M., 1997. Sources of ion outflow in the high latitude ionosphere. Space Science Reviews 80, 1. 6 8 9 
2

3

4

5

6

7

8

9

10

11

12

13

14

15

16

17

18

19

20

21

22

\section{Solar Wind Parameters}

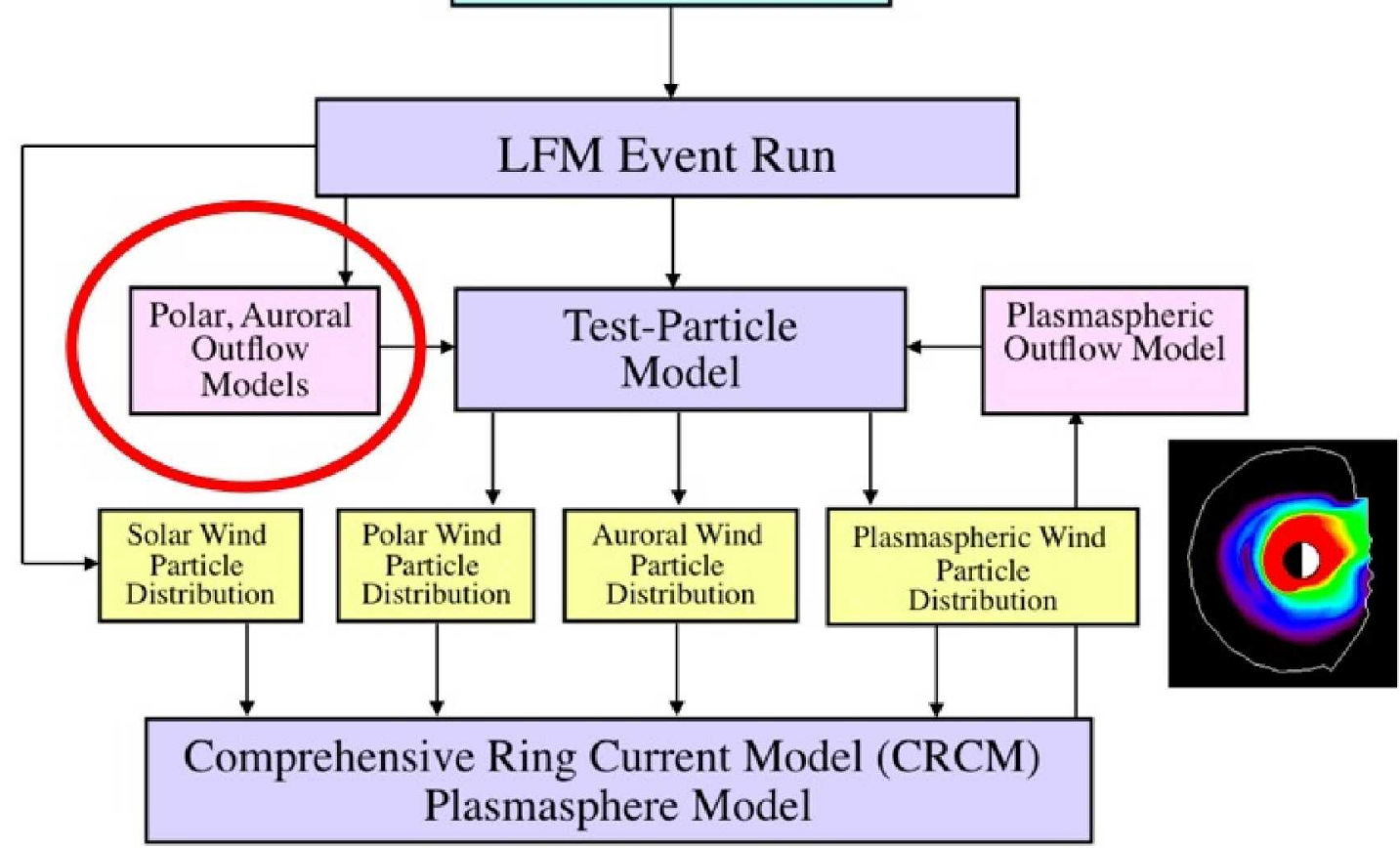

Figure 1. Schematic diagram of the models used to simulate the featured event. 
Figure 2. WIND observations of the solar wind conditions for a 10-hour period on 24-25 [2006]. 
2

3

4

5

6

7

8

9

10

11

12
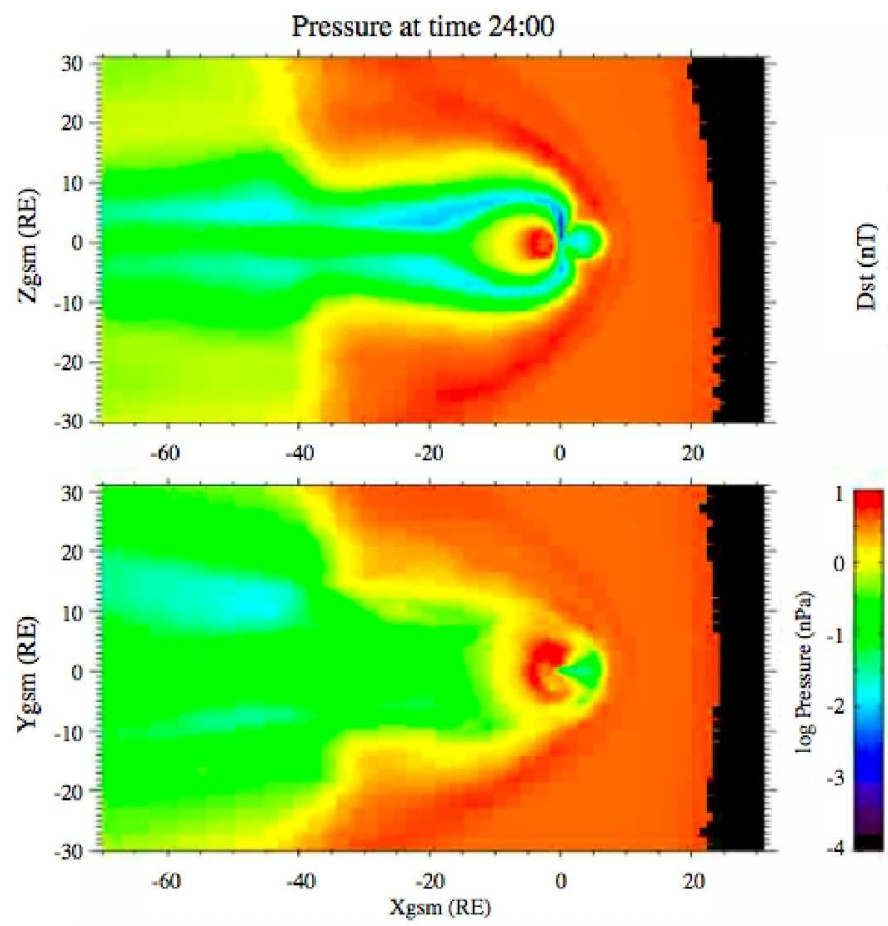
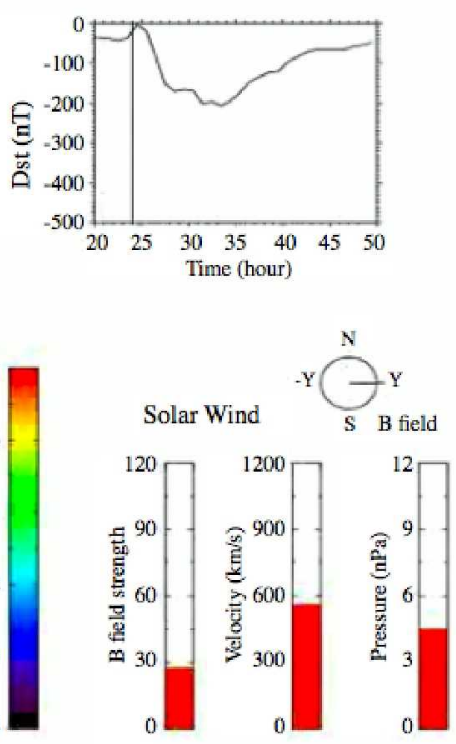

Figure 3. Illustration of the above described conditions shortly after CME arrival, with the shock traveling down the magnetotail, as modeled by the LFM global simulation. Times are relative to 00 UT on 24 Sep 1998. The midnight near-Earth plasma sheet has a region of pressure comparable to that of the solar wind extending across it nearly from flank to flank. 
2

3

4

5

6

7

8

9

10

11

12
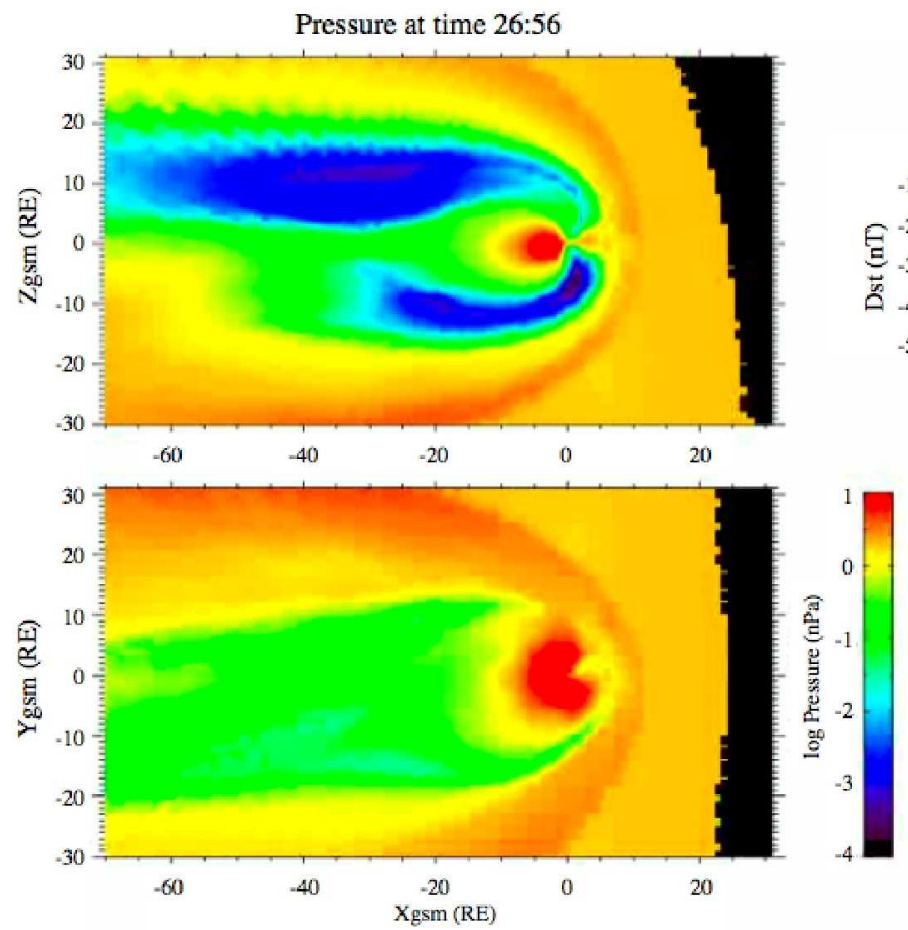
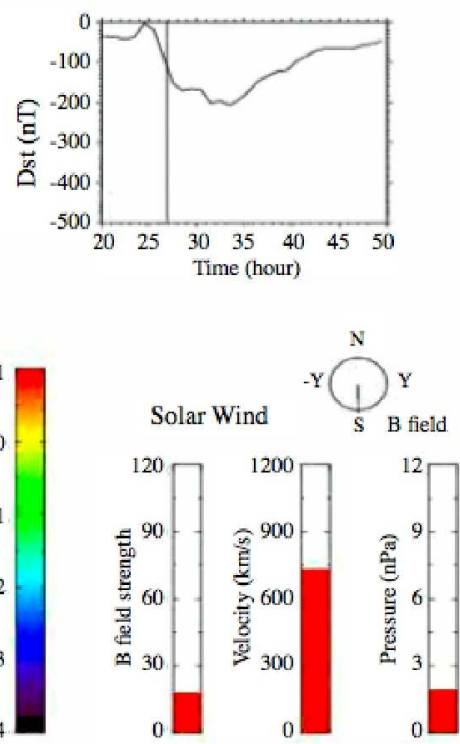

13

14

Figure 4. Solar wind conditions and magnetospheric configuration at the peak rate of 15 growth of the ring current.

16

17

18

19

20

21

22 
1998 Sep 24-25 Polar with Caps 5 million Particles

3

4

5

6

7

8

9

10

11



$X=0$ plane

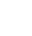

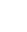
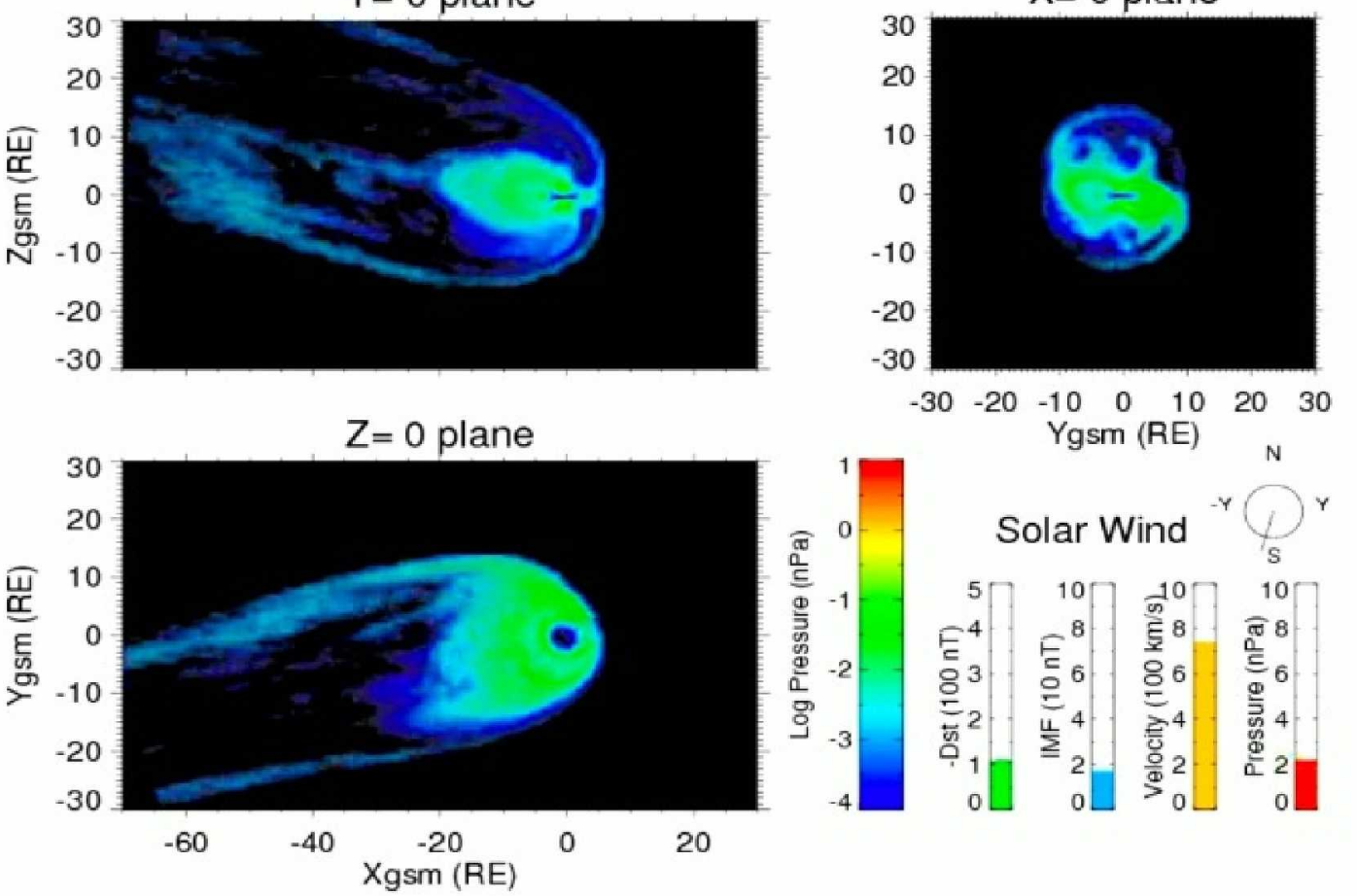

12

Figure 5. Solar wind conditions and resultant simulated polar wind circulation at the peak

14 rate of growth of the ring current, within the LFM simulated fields.

15

16

17

18

19

20

21 
2

3

4

5

6

7

8

9

10

11

12

13

14

15

16

17

18

19

20

21

22

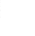

8



Frame 170.1 Time 30:59.3 UT

hot $\mathrm{Ne}$

Log Density

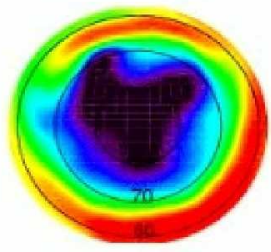

10

1

Log Flux



Sep98 North MHD Conditions

Log Poynting Flux
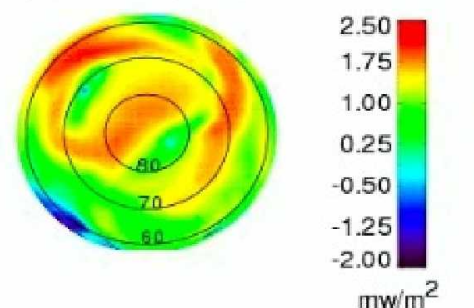

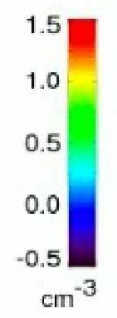

Auroral Wind Outflow Parameters
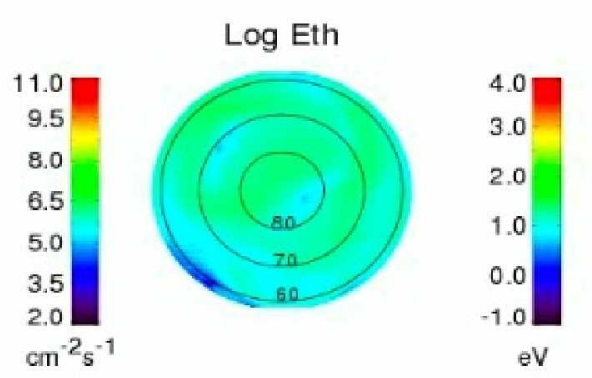
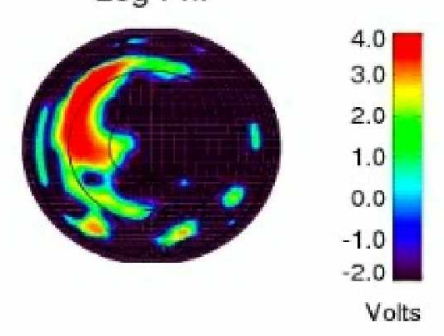

Ver 12

$V_{/ /}$to $B$ Log Phi

$=$

Volts

Log E Parallel
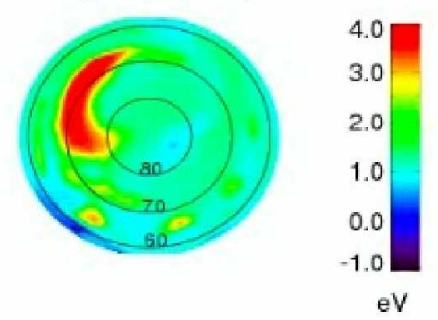

Figure 6. Snapshot at indicated time of ionospheric polar distributions of: (upper row drivers) MHD precipitating plasma density, DC Poynting flux at $4000 \mathrm{~km}$ altitude, and parallel potential drop from parallel current density; (lower row outflowing $\mathrm{O}^{+}$ion properties): $\mathrm{O}^{+}$escape flux, thermal energy, and parallel energy (sum of $\mathrm{E}_{\mathrm{th}}$ and $\mathrm{eV}_{/ /}$). 
2

3

4

5

6

7

8

9

10

11

12

13

14

15

16

17

18

19

20

21

22

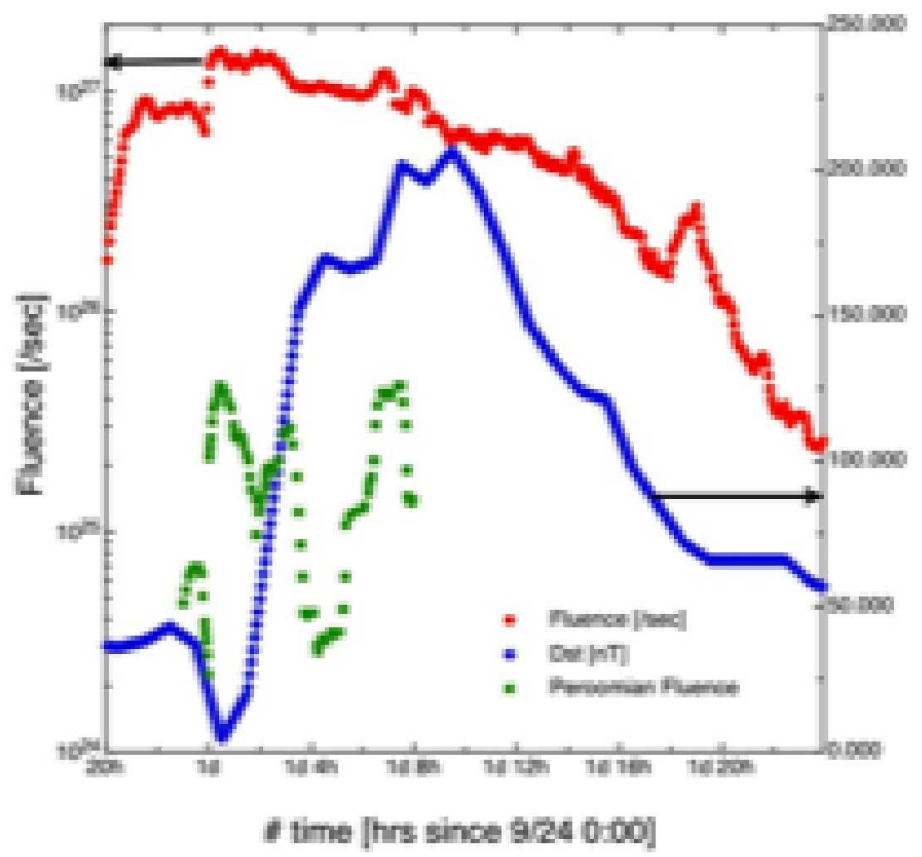

Figure 7. Temporal development of simulated auroral wind outflow fluence (left axis) computed for the 24-25 September 1998 simulation period by this study and by Peroomian et al. [2006], compared with $\mathrm{D}_{\mathrm{ST}}$ (inverted, right axis). 


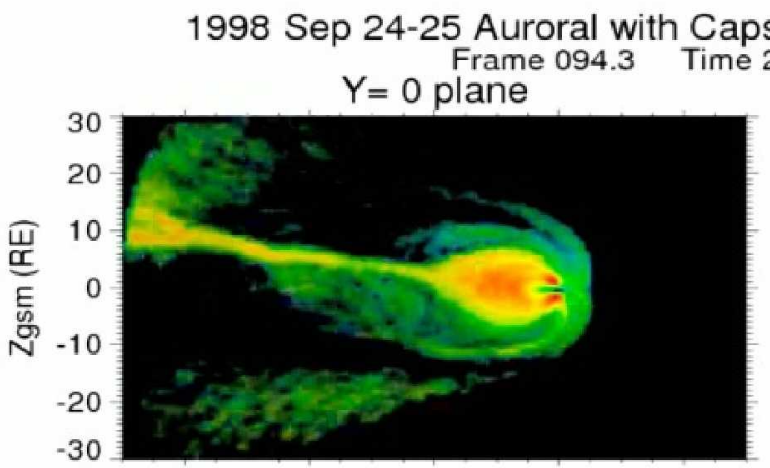

$3,000,000$ Particles

3

4

5

6

7
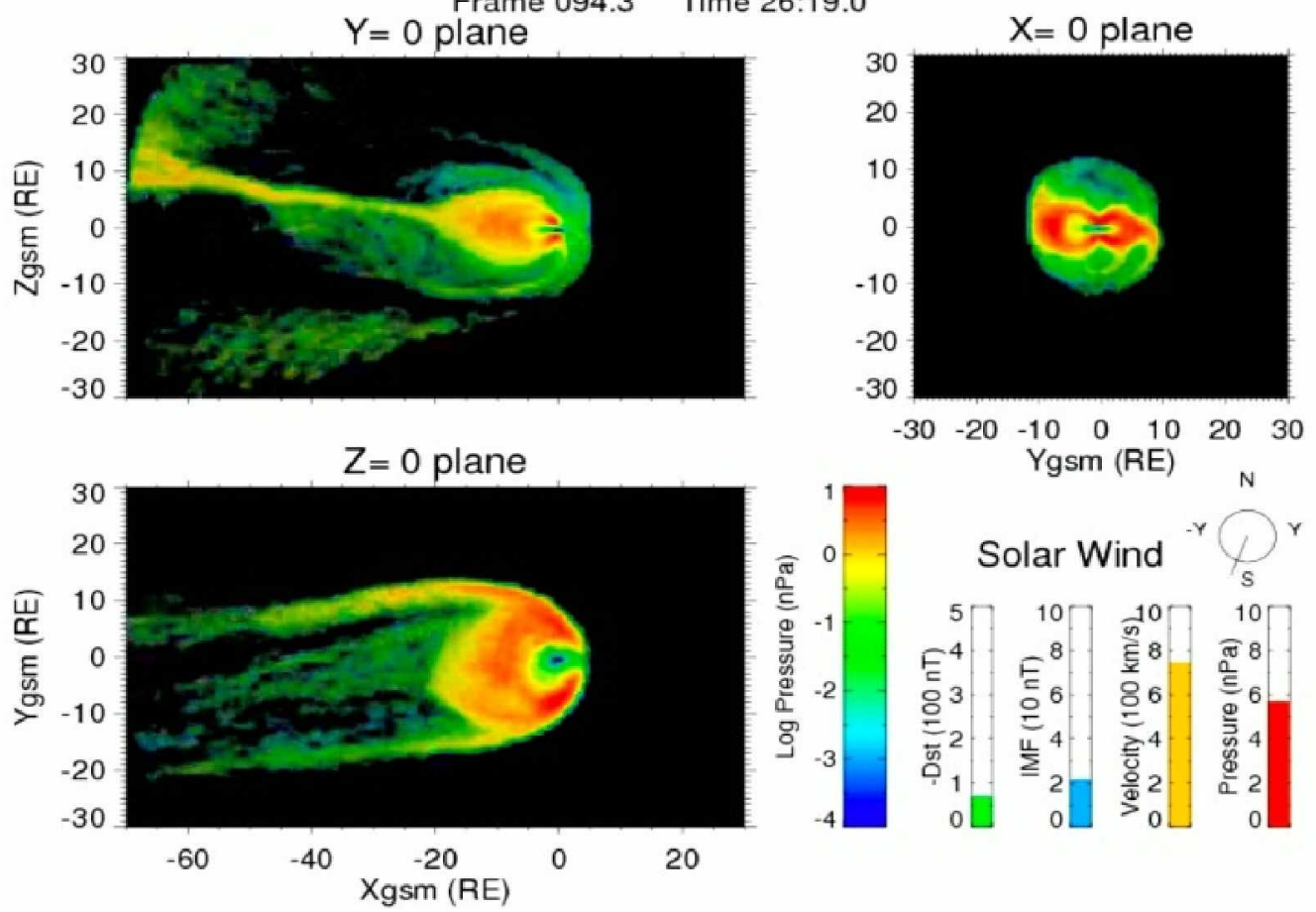

8

9

10

11
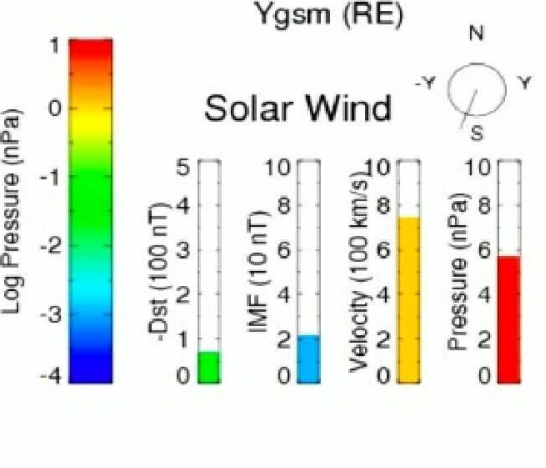

12

Figure 8. Simulated distribution of $\mathrm{O}^{+}$ion pressure during the simulated storm growth

14 phase. 

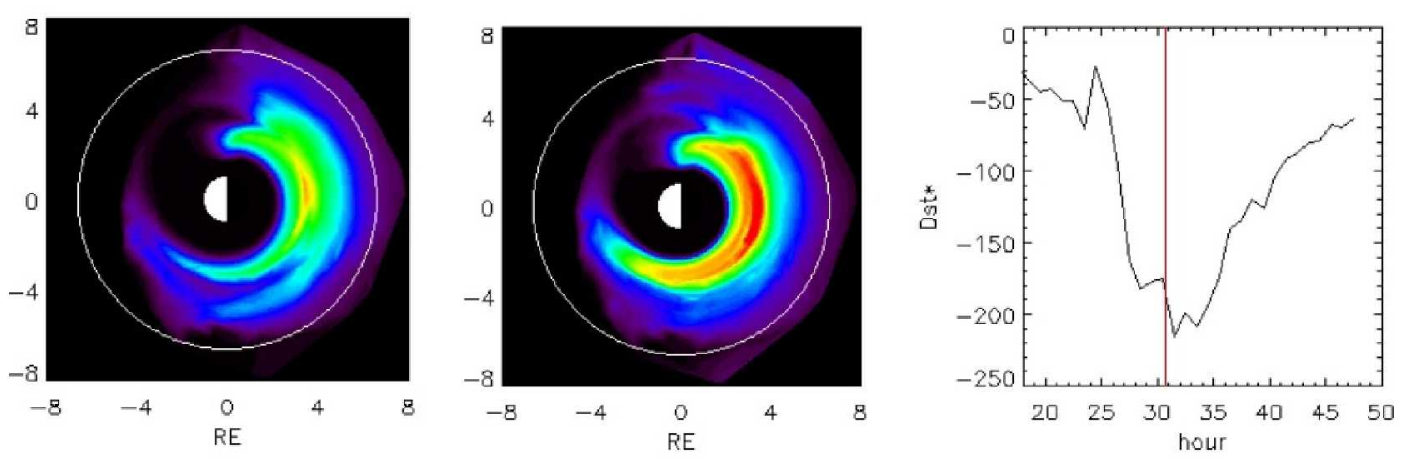

8

pressure ( $\mathrm{nPg}$ )

prossure $(\mathrm{nPa})$


Figure 9. Inner magnetospheric pressure distribution in the $\mathrm{XY}$ plane for the two principal components, ionospheric $\mathrm{O}^{+}$and solar wind $\mathrm{H}^{+}$, shown near the peak of the 12 storm epoch at 30:40 UT after 00:00 of 24 Sept. 1998, as simulated using the GIK formalism to drive the CRCM component of the code.

14 


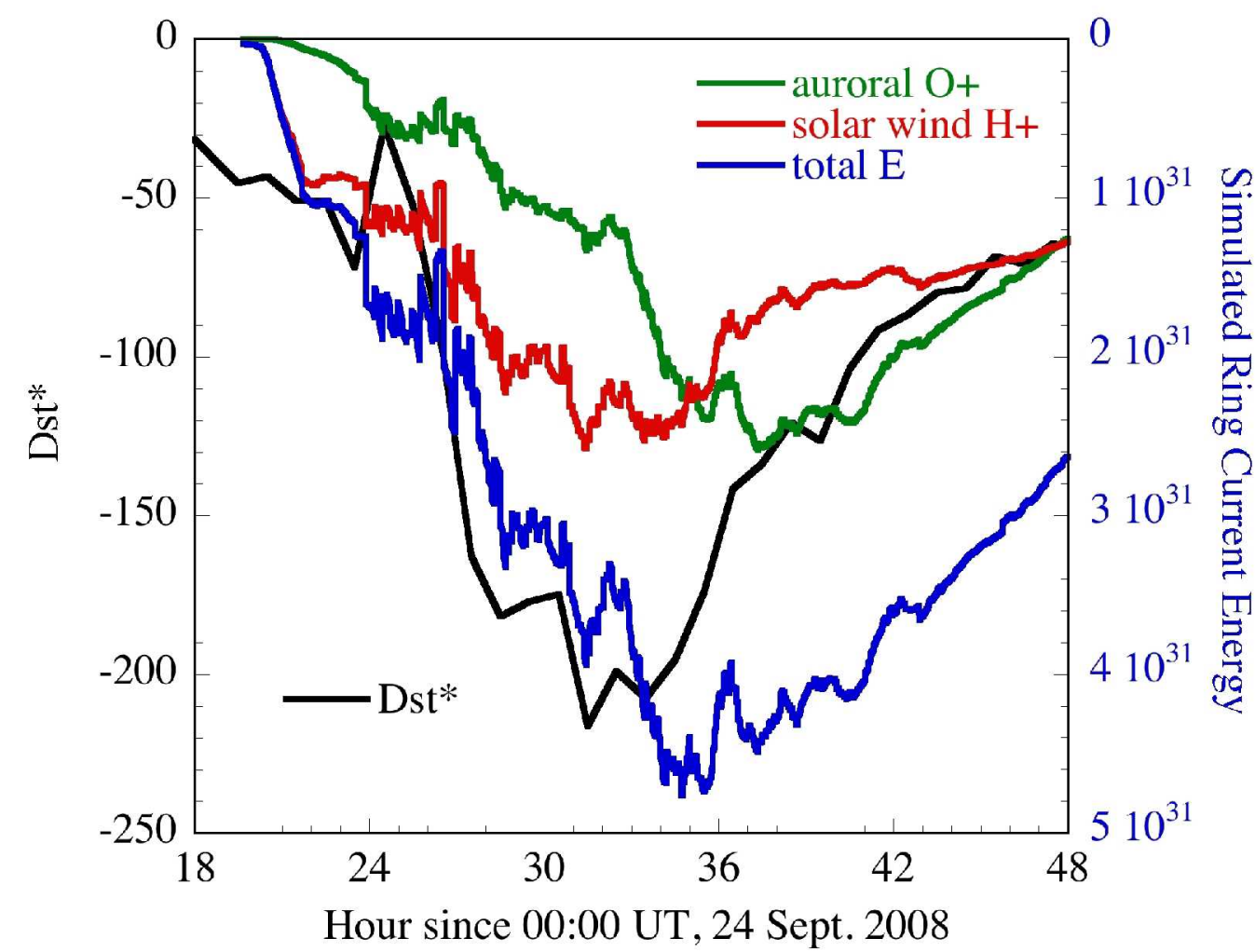

13

Figure 10. Total energy content for solar and auroral wind plasmas, compared with Dst 15 development for the simulated period.

16

17

18

19

20

21

22 